\title{
III. R\&A als Bestandteil der amerikanischen Kriegsmaschinerie
}

\section{Geistes- und sozialwissenschaftliche Expertise im Dienste militärischer Operationen}

Es ist behauptet worden, daß in Washington Einigkeit darüber bestand, "[that] R\&A ought not to have to be created: everything it would do should have been done before the war began in Europe ${ }^{\text {"1 }}$. Wenn auch zu bezweifeln ist, daß die Einmütigkeit in diesem Punkte tatsächlich vollständig und ungebrochen war, so hatte die R\&A-Branch doch frühzeitig ihre Nützlichkeit unter Beweis stellen können ${ }^{2}$ und war zudem von Anfang an die am wenigsten kontroverse COI-Abteilung. Zum Teil lag das daran, daß Donovan die meisten anderen Aufgabenbereiche, die er für seine Behörde erschlieBen wollte, gegen den Widerstand der bereits etablierten Nachrichtendienststellen als COI-Kompetenz reklamieren mußte, wobei bald deutlich wurde, "[that] the policy of cloaking in vagueness the true purposes of COI in the authorizing order of $11 \mathrm{July}$ was beginning to boomerang [...] inevitably COI began to come into real or imagined conflict with other departments and agencies"3.

Seine gewichtigsten Konkurrenten hatte Donovan selbst schon in seinem „Memorandum of Establishment of Service of Strategic Information" vom 10.Juni 1941 identifiziert: das FBI und die Nachrichtendienststellen der Armee (G-2) und der Marine (ONI). Aber auch State und War Department wachten eifersüchtig über ihre Privilegien; hinzu kam die erbitterte Konkurrenz des Coordinator of InterAmerican Affairs, Nelson Rockefeller, sowie die solch neuer Einrichtungen wie des Office of Civilian Defense, des Board of Economic Warfare und des Office of War Information. Nicht wenige der Kämpfe um Kompetenzen und Domänen, die Donovan auszufechten hatte, gingen zu seinen Ungunsten aus ${ }^{4}$. Dabei spielten nicht nur die Beziehungen der jeweils involvierten Persönlichkeiten zu einflußreichen Kreisen um den Präsidenten eine Rolle - über die verfügte Donovan schließlich ebenfalls ${ }^{5}$-, sondern auch das Urteil des Bureau of the Budget, und dieses zeigte sich „increasingly concerned with the contrast between COI as planned and COI as developing "6.

\footnotetext{
1 Winks, Cloak, S.71; dieser Gedanke ist allerdings der R\&A-History entnommen, vgl. NA, RG 226, R\&A-History, S.1.

2 Vgl. dazu auch OSS War Report I, S. 17.

${ }^{3}$ Ebenda, S.15.

4 Einzelheiten bei Troy, Donovan, S. 94-102, $118 \mathrm{ff}$.

${ }^{5}$ Donovan hatte z.B. offenbar gute persönliche Beziehungen zum Secretary of the Navy, Frank Knox, und mit gewissen Einschränkungen auch zum Secretary of War, Stimson. Sein Einfluß auf Roosevelt ist allerdings besonders in den Donovan-Biographien sowie in den von ehemaligen OSS-Mitarbeitern verfaßten Werken immer wieder überschätzt worden, vgl. dagegen Troy, Donovan, S.116, sowie Smith, Shadow Warriors, S.30ff., und OSS War Report I, S.20.

6 Troy, Donovan, S. 100; ähnlich OSS War Report I, S. 20.
} 
Denn ungeachtet der eben angedeuteten Konflikte, zu denen auch noch Unterbringungsschwierigkeiten und administrative Probleme kamen, wuchs Donovans Behörde fast täglich und entwickelte sich in der Tat in vorher kaum abzusehenden Dimensionen?. Das Bureau of the Budget, dessen Aufgabe es war, darauf zu achten, daß nicht zwei Behörden für ein und dieselbe Tätigkeit Mittel in Anspruch nahmen, hatte die ungeahnte Expansion des COI von Anfang an mit Mißtrauen beobachtet und bereits Ende August 1941 „functional confusion " ${ }^{8}$ innerhalb des kaum zwei Monate bestehenden COI konstatiert. Dieses Urteil entsprang sicherlich nicht nur bürokratischer Mißgunst. Wenn der COI bzw. das aus ihm hervorgehende Office of Strategic Services in den Erinnerungen ehemaliger Mitarbeiter immer wieder als „a direct reflection of Donovan's character ${ }^{49}$ beschrieben wird, dann beinhaltete dies nicht nur Bewunderung für Donovans unermüdlichen Einsatz, sondern auch eine mehr oder minder sentimentale Kritik an seinem vollständigen Desinteresse für organisatorische und administrative Probleme. Ebenso wie seine bereits angesprochene, nie nachlassende Begeisterungsfähigkeit für neue Projekte - und wenn sie noch so exotisch anmuten mochten - prägte diese Eigenheit Donovans zweifellos den Charakter der von ihm geleiteten Behörde ${ }^{10}$.

In dem durch die Schaffung des COI in Washington wieder einmal entbrannten „dog-eat-dog struggle among government departments "11 schien Donovans Behörde so eine leichte Beute zu sein, und schon Anfang 1942 begann sich abzuzeichnen, daß das Weiterbestehen des COI in der Tat gefährdet war. Zwar konnte sich Donovan darauf berufen, daß seine Behörde bislang zur vollen Zufriedenheit Roosevelts gearbeitet hatte ${ }^{12}$, doch reichte der sowieso nicht allzu engagierte Schutz durch einen vielbeschäftigten Präsidenten nicht aus, um das Bestehen des COI im Grabenkampf der Washingtoner Bürokratien auf lange Sicht zu sichern. Der Krieg hatte zudem zahlreiche neue Behörden und Dienststellen aus dem Boden schießen lassen, die ebenfalls mit der Sammlung, Verarbeitung und Verbreitung von Nachrichten befaßt waren und die Konkurrenz auf diesem Gebiet noch verschärften ${ }^{13}$.

Am drastischsten sollte sich die Washingtoner Szenerie schließlich durch den japanischen Überraschungsangriff auf Pearl Harbor am 7. Dezember 1941 verändern ${ }^{14}$ : Die Vereinigten Staaten waren damit unweigerlich in den Krieg verwickelt, und isolationi-

7 Ausführliche Darstellung der Expansion des COI in den ersten sechs Monaten seines Bestehens bei Troy, Donovan, S. 73-116. Nach etwa einem Jahr umfaßte der COI ungefähr 2000 Mitarbeiter und verfügte zusätzlich über Außenposten in London, Cairo und Chungking, vgl. OSS War Report I, S.26.

${ }^{8}$ Troy, Donovan, S.110; zur kritischen Haltung des Bureau of the Budget gegenüber dem COI siehe auch OSS War Report I, S. $20 \mathrm{f}$.

9 Alsop/Braden, Sub Rosa, S.10f.; es gibt aber viele ähnliche Äußerungen in der von ehemaligen OSS-Mitarbeitern verfaßten Literatur, und nicht selten wird Donovans Bedeutung für den Aufbau des OSS geradezu als symbiotisch beschrieben, vgl. z. B. Alcorn, No Bugles, S. 36; siehe auch OSS War Report I, S.29. Selbst Cave Brown gesteht zu - nachdem er Donovan als „queer figure who comes off three-quarters Machiavelli and one-quarter boy" charakterisiert: „yet, to be fair, Donovan was the OSS and the OSS was Donovan, at least at first." Cave Brown, Last Hero, S. $5 \mathrm{f}$.

${ }^{10}$ Siehe dazu Smith, OSS, S. 1-35: „Donovan's Dreamers“.

${ }_{11}$ So Ford, Donovan, S. 109.

${ }^{12}$ Dazu ausführlich Troy, Donovan, S.111-116.

${ }_{13} \mathrm{Vgl}$. dazu den Überblick ebenda, S.209f.

14 Der noch im Aufbau befindliche COI war zu diesem Zeitpunkt in keiner Weise in einer Position, die es ihm erlaubt hätte, den japanischen Angriff vorauszusehen; in der einschlägigen Lite- 
stische Zweifel machten einer erbitterten Entschlossenheit Platz, die dem amerikanischen Volk angetane „Infamie“ wettzumachen. Im Konkurrenzkampf der Washingtoner Bürokratien hatte das Militär nun eindeutig die Oberhand, was für den COI bedeutete, daß die Billigung seiner Aktivitäten durch das Weiße Haus allein nicht mehr ausreichen konnte. Vor diesem Hintergrund entwickelte sich Anfang 1942 in Washington eine hitzige Debatte über eine Reorganisation des Nachrichtenwesens, in der, wie im OSS War Report etwas säuerlich bemerkt wird, „many in Washington who did not believe they knew exactly what should be done, at least thought they knew what someone else should be prevented from doing "15. Im Rahmen dieser mit entsprechender Erbitterung geführten Debatte zirkulierten bald auch Vorschläge, die die Abschaffung des COI bzw. seine Aufteilung unter bereits bestehende Behörden ins Auge faßten $^{16}$.

Daß der COI diese Reorganisationsdebatte relativ unbeschädigt als Office of Strategic Services (OSS) und als „supporting agency“ der Joint Chiefs of Staff (JCS), also der nach britischem Vorbild organisierten militärischen Führungsspitze der US-Streitkräfte überstand, war nicht ohne Ironie. Schließlich hatten die Militärs ihr von Anfang an bestehendes Mißtrauen gegen Donovans Behörde nie aufgegeben und sie stets als eine Art Sicherheitsrisiko betrachtet. Insofern waren sie aber auch daran interessiert, den COI unter Kontrolle zu bekommen. Das galt um so mehr, als die US-Militärs, die bislang nicht viel von „subversive activities“ und „psychological warfare“ gehalten hatten, seit ARCADIA, der britisch-amerikanischen Konferenz während der Jahreswende 1941/ 42, gezwungen waren, sich mit diesem Thema auseinanderzusetzen. Denn während dieser Konferenz war eine Einigung über eine gemeinsame amerikanisch-britische "Grand Strategy" zustande gekommen, wobei die Briten den Standpunkt vertreten hatten, daß "subversive activities“ integraler Bestandteil der Kriegführung zu sein hätten $^{17}$. Vor diesem Hintergrund begannen die JCS, sich ernsthaft für Donovans diesbezügliche Pläne und Vorarbeiten zu interessieren und eine Aufteilung des COI auf verschiedene Abteilungen des Militärs zu erwägen. Eine solche Lösung konnte freilich kaum im Sinne Donovans sein, selbst wenn angesichts des Kriegseintritts der Vereinigten Staaten eine engere Bindung an das Militär und vor allem an dessen Ressourcen durchaus Vorteile versprach. Deshalb stand Donovan einer Unterstellung seiner Behörde unter die JCS keineswegs grundsätzlich ablehnend gegenüber, wobei ihm allerdings daran gelegen war, den COI als Einheit zu bewahren ${ }^{18}$. Die Lösung, die letztendlich zustande kam, entsprach insofern nur bedingt Donovans Vorstellungen: Die „Military

ratur ist der Überraschungsangriff auf Pearl Harbor jedoch ein vielzitiertes und vieluntersuchtes Beispiel für das Versagen der amerikanischen Nachrichtendienste, vgl. z. B. die Arbeiten von Prange, insbesondere: Pearl Harbor: The Verdict of History.

15 OSS War Report I, S. 19.

${ }^{16}$ Detaillierte Darstellung der Hintergründe und Zusammenhänge bei Troy, Donovan, S. $120 \mathrm{ff}$., vgl. auch S. $209 \mathrm{ff}$.; sowie OSS War Report I, S.19ff.

17 Vgl. dazu und zum Folgenden Troy, Donovan, S.129ff.; sowie OSS War Report I, S.21; zur ebenfalls erst rudimentär entwickelten britischen Expertise in "shadow warfare" siehe Smith, Shadow Warriors, S.3-54; sowie die Entstehungsgeschichte der britischen SOE bei Stafford, Britain and the European Resistance, S.10-49.

$18 \mathrm{Vgl}$. dazu auch die in OSS War Report I, S. 24 abgedruckten Schreiben Donovans an Roosevelt vom Frühjahr 1942, sowie FDRL, PSF/Subject, b 165, f 7: Donovan, Memorandum for the President, 16.3. 1942. 
Order" vom 13. Juni 1942 bestimmte, daß der COI, umbenannt in Office of Strategic Services, und seiner Funktionen im Bereich „foreign information“ enthoben ${ }^{19}$, der Kontrolle der JCS unterstellt wurde ${ }^{20}$. Der Kampf um Kompetenzen und Aufgabenbereiche sollte damit für Donovans Behörde allerdings noch immer nicht beendet sein, und entsprechend düster schließt der OSS War Report seine Zusammenfassung der Entwicklung des COI zum OSS ab: „The period of uncertainty as to the disposition of COI by the President gave way to a new period of uncertainty as to the future of OSS under the Joint Chiefs of Staff. “21

Zunächst erschien es sogar fraglich, ob das OSS nicht letztlich völlig im militärischen Apparat aufgehen würde. Erst nach monatelangem bürokratischen Tauziehen gelang Donovans Behörde Ende 1942 endlich der Durchbruch"22: Die Direktive JCS 155/4/D vom 23. Dezember 1942 definierte erstmals in umfassender Weise die Aufgaben und Kompetenzen des OSS $^{23}$. Diese bestanden demnach zum einen in "planning, development, coordination, and execution of the military program for psychological warfare“, wobei nicht näher ausgeführt wurde, was genau unter „psychological warfare“ zu verstehen sei $^{24}$. Es wurde lediglich festgelegt, daß das OSS in Erfüllung dieser Aufgaben für "special operations“ wie Sabotage, Spionage und Gegenspionage in feindlich besetzten Gebieten sowie für den Kontakt mit Untergrundbewegungen und für die Organisation und Führung von Guerillakrieg zuständig sei. Darüber hinaus wurde das OSS beauftragt, „[to compile] such political, psychological, sociological, and economic information as may be required for military operations". Dies umschrieb den

19 Die Propaganda-Abteilung des COI, der unter Leitung von Robert E. Sherwood stehende Foreign Information Service (FIS), war, wie bereits erwähnt, von Anfang an die problematischste COI-Abteilung gewesen. Die Executive Order 9182 vom 13.6. 1942, durch die - gleichzeitig mit Unterstellung des COI/OSS unter die JCS - das Office of War Information eingerichtet wurde, verfügte die Eingliederung des FIS in diese neugegründete Behörde, vgl. Executive Order 9182, abgedruckt bei Troy, Donovan, S. 424-426. Die Abtrennung des FIS war kaum in Donovans Sinne, vgl. OSS War Report I, S.21 ff.

${ }^{20}$ Die Military Order vom 13.6. 1942 „Office of Strategic Services“ ist abgedruckt bei Troy, Donovan, S.427; sowie bei Corson, Armies of Ignorance, S. 187; vgl. auch JCS 67 vom 21.6. 1942, abgedruckt bei Troy, Donovan, S.428. Als diese Direktive herausgegeben wurde, war Donovan gerade in London, und offensichtlich war er nicht davon informiert worden, daß eine derartige Regelung unmittelbar bevorstand, vgl. OSS War Report I, S.26, und Troy, Donovan, S. $150 \mathrm{ff}$.

${ }^{21}$ OSS War Report I, S.27.

22 Ausführliche Darstellung der Hintergründe und Zusammenhänge bei Troy, Donovan, S. 155191; vgl. auch Smith, Shadow Warriors, S.140ff., sowie OSS War Report I, S. 97 ff.

${ }^{23} \mathrm{JCS} 155 / 4 / \mathrm{D}$ ist abgedruckt bei Troy, Donovan, S. 431-434; letztlich sollten aber noch mehrere Direktiven notwendig sein, bis dem OSS schließlich durch die Direktive JCS 155/11/D vom 27.10. 1943 "definite authorization and sufficient scope to develop maximum efficiency" verschafft wurden, vgl. OSS War Report I, S. 105-110, und NA, RG 226, R\&A-History II, S.11, sowie Troy, Donovan, S.191 ff. JCS 155/11/D ist abgedruckt ebenda, S.439ff.; diese Direktive definierte erstmals auch umfassend die Aufgaben von R\&A.

${ }^{24}$ Erst sehr viel später wurde erkannt, daß die fortgesetzte Verwendung dieses ungeklärten Begriffs zur Beschreibung eines wesentlichen Aufgabenbereiches des OSS viel Verwirrung stiften mußte, siehe dazu Troy, Donovan, S.206, sowie Donovan an Roosevelt, Schreiben vom 23.2. 1943, in: FDRL, PSF/Subject, b 167; im März 1943 wurde schließlich in einer R\&A-Denkschrift versucht, eine umfassende Definition vorzulegen, in: NA, RG 226, E 37, b 5, f: Psychological Warfare Committee - Research and Analysis. 
Aufgabenbereich der R\&A-Branch, deren Arbeit - wie auch die übrigen Dienstleistungen, die das OSS zu erbringen hatte - nun stets den vom Militär definierten Erfordernissen und Notwendigkeiten Rechnung zu tragen hatte. Das war schließlich der Preis, den Donovans Behörde für ihre Eingliederung in den machtvollen und immer mehr expandierenden Apparat der JCS zu entrichten hatte.

Während einige OSS-Abteilungen von dieser Entwicklung durchaus profitierten, wirkte sie sich für R\&A eher negativ aus. Wie bereits erwähnt, hatte die R\&A-Branch die ihr von Donovan ursprünglich zugedachte zentrale Stellung schon im Zuge der Expansion des COI einzubüßen begonnen. Durch die Eingliederung des OSS in den unter Kontrolle der JCS stehenden militärischen Apparat wurde diese Tendenz in gewissem Sinne verstärkt. Denn da das OSS nun in erster Linie mit der Entwicklung und Umsetzung irregulärer Kampfstrategien beauftragt war, traten die „operational branches“ wie etwa die mit Sabotageaktionen und Guerrillakampf befaßten Special Operations (SO) oder die für „schwarze“ Propaganda und Subversion zuständige Moral Operations-Abteilung (MO) zunehmend in den Vordergrund; ebenso gewannen die mit Spionage befaßte Secret Intelligence-Abteilung (SI) sowie die für Abwehr verantwortliche X-2-Abteilung in diesem Zusammenhang erheblich an Gewicht.

Innerhalb des OSS entwickelte sich die R\&A-Branch so in zunehmendem Maße zu einer Dienstleistungsabteilung. Auch wenn die durch R\&A zugänglich gemachten Informationen sowie die dort ausgearbeiteten Studien und Analysen letztlich oft genug die unverzichtbare Grundlage für die Arbeit der anderen OSS-Abteilungen bildeten, war damit ein gewisser Prestigeverlust verbunden, zumal diejenigen OSS-Mitarbeiter, die per Schlauchboot oder Fallschirm in feindlich besetzte Gebiete eindrangen oder derartige Aktionen wenigstens am Schreibtisch erdachten, der soviel weniger spektakulären Arbeit ihrer Kollegen vom R\&A- „Campus“ nicht immer den verdienten Respekt entgegenbrachten. Dabei ist immer wieder darauf hingewiesen worden, daß auBerhalb des OSS stets Einigkeit darüber bestand, daß die R\&A-Branch, die schließlich weiterhin für das Außenministerium und für militärische Stellen sowie zahlreiche Regierungsbehörden arbeitete, die bedeutendste OSS-Abteilung darstellte ${ }^{25}$. Auf seiten des Militärs hatte man den Wert der von R\&A geleisteten Arbeit schon früh erkannt $^{26}$, und im März 1942 war in einem von den JCS in Auftrag gegebenen Bericht zur Frage einer eventuellen Aufteilung des COI gar zu lesen, die R\&A-Branch sei „a school of advanced research of the highest caliber which can readily be used effective$1 y^{* 27}$.

Bald nach der Übernahme des OSS durch die JCS hatten die R\&A-Mitarbeiter Gelegenheit zu zeigen, daß dieses Urteil gerechtfertigt war. Denn im Rahmen der Vorbereitungen zu der zwischen Roosevelt und Churchill vereinbarten alliierten Landung in Nordafrika, die unter dem Codenamen TORCH für November 1942 geplant war, wurde die Ausarbeitung von umfassenden Studien über die topographi-

${ }^{25}$ So z. B. Winks, Cloak, S.61, 114; Corson, Armies of Ignorance, S. 168; vgl. auch OSS War Report I, S.167. Vgl. außerdem die bei Katz, Foreign Intelligence, im Anhang wiedergegebene Graphik „Flow of R\&A Intelligence Material“ (Chart 3).

${ }^{26}$ Dazu OSS War Report I, S. 17.

${ }^{27}$ Zit. nach Troy, Donovan, S. 133. Dem Bericht zufolge verfügte R\&A zu diesem Zeitpunkt über 340 Mitarbeiter (darunter 150 Wissenschaftler) und einen Etat von \$1 Million. 
schen, geographischen, politischen und wirtschaftlichen Gegebenheiten in Marokko, Algerien und Tunesien notwendig ${ }^{28}$. Der COI selbst war in Nordafrika schon seit Januar 1942 aktiv $^{29}$. Unter der Leitung von Colonel William Eddy war ein geheimes Radionetz an der nordafrikanischen Küste aufgebaut und Kontakte zu französischen Anti-Vichy-Gruppierungen sowie zu einheimischen Gruppen geknüpft worden. Da sich der Widerstand der letzteren jedoch zumeist nicht nur gegen eine deutsche Besatzung, sondern ebenso gegen die französische Kolonialherrschaft richtete, wurde ihre vom COI angeregte Einbeziehung als Guerillakampfverbände in das geplante alliierte Landungsunternehmen aufgrund von Bedenken im Außenministerium sowie in militärischen Kreisen bald verworfen ${ }^{30}$. Der herausragende Beitrag des COI bzw. des OSS zur alliierten Landung in Nordafrika bestand dann auch keineswegs in der Planung und Umsetzung irregulärer Kampfstrategien. Es waren vielmehr die von Eddy und seinen Mitarbeitern nach Washington und London - dem Standort von General Eisenhowers Allied Forces Headquarter - übermittelten strategisch wichtigen Informationen und Daten sowie die hauptsächlich von der unter Leitung von Sherman Kent stehenden Mediterranean and Africa Section ausgearbeiteten R\&AStudien, die dem OSS die Anerkennung der Militärs verschafften und seine Nützlichkeit für die Vorbereitung und Ausführung militärischer Operationen unter Beweis stellten.

Der Herausgeber des OSS War Report, Kermit Roosevelt, sah später im OSS-Beitrag zur Operation TORCH gar den Hauptgrund für das „Überleben“ des OSS: „Without this evidence to the JCS of its value, it would most likely have been dismembered. " ${ }^{11}$ Im Urteil Donovans bedeuteten die von der Mediterranean and Africa Section in Marathon-Arbeitsgängen erstellten umfassenden Nordafrikastudien darüber hinaus "the first victory “ für die Arbeitsweise der R\&A-Branch ${ }^{32}$.

\section{Interdisziplinäres Forschen für Krieg und Frieden}

Obwohl die von R\&A bei der Vorbereitung der alliierten Landung in Nordafrika geleistete Arbeit allgemein Anerkennung gefunden hatte, war man sich in der Abteilung längst darüber im klaren, daß die wenig durchdachte interne Struktur und die ineffiziente Verwaltung der Abteilung ihre Leistungsfähigkeit beeinträchtigten ${ }^{33}$. Zum einen war die Aufteilung in die nach regionalen Schwerpunkten gegliederte Division of Spe-

${ }^{28}$ Vgl. dazu Breuer, Operation Torch.

${ }^{29}$ Dazu Smith, OSS, S. $41 \mathrm{ff}$.; sowie Smith, Shadow Warriors, S. $146 \mathrm{ff}$.; OSS War Report I, S. $93 \mathrm{ff}$. und II, S. 9 ff.; vgl. auch Koch, Der amerikanische Geheimdienst OSS, in: Schulz (Hrsg.), Geheimdienste und Widerstandsbewegungen, S.79-104, insbesondere S. $84 \mathrm{ff}$., sowie Walker, OSS and Operation Torch, in: JCH 22 (Oktober 1987), S.667-679.

${ }^{30} \mathrm{Vgl}$. dazu jedoch Smith, OSS, S. $41 \mathrm{ff}$.

${ }^{31}$ OSS War Report I, Introduction x; vgl. dazu aber die kritische Bewertung bei Walker, OSS and Operation Torch, in: JCH 22 (Oktober 1987), insbesondere S. $674 \mathrm{ff}$.

32 Zit. nach Winks, Cloak, S. 85; eine farbige Darstellung der Umstände, unter denen die Nordafrikastudien entstanden, ebenda, S. 84 f.; vgl. auch NA, RG 226, R\&A-History (IV. EuropeAfrica Division), S. 14.

${ }^{33}$ NA, RG 226, R\&A-History (II), S. 15. 
cial Information (DSI) und die funktionalen Abteilungen für Psychology, Economics und Geographics kaum geeignet, die Flexibilität und Kooperation innerhalb der Abteilung zu gewährleisten, die die Bandbreite und Eigenart der von R\&A bearbeiteten Themen und Fragestellungen erforderten ${ }^{34}$. Abteilungsinterne Probleme wurden zudem dadurch verschärft, daß sich R\&A-Direktor Baxter wenig um Verwaltungsfragen kümmerte, da er vollständig davon in Anspruch genommen war, seiner Abteilung einen festen Aufgabenkreis innerhalb des COI bzw. des OSS und einen „Kundenstamm" in der Regierungsbürokratie zu sichern ${ }^{35}$.

Diese Vernachlässigung interner Administration und die problematische Struktur der Abteilung führten bald zu starken Spannungen, deren Lösung sich Baxter wohl nicht gewachsen fühlte, und im Herbst 1942 trat er schließlich als Leiter der R\&ABranch zurück. Es war bezeichnend für Donovans Führungsstil - „throwing power into the middle of his subordinates and letting the strongest emerge on top ${ }^{436}-$, daß er die Nachfolge Baxters zunächst offenließ. William Langer hatte jedoch wohl schon bei seiner Einstellung im Sommer 1941 damit gerechnet, die Leitung von R\&A zu übernehmen, und zögerte nach Baxters Rücktritt offenbar nicht, de facto dessen Nachfolge anzutreten. Enthusiastisch unterstützt von zwei jungen Assistenten, Carl Schorske und Robert Wolff, begann Langer unverzüglich, den Aufbau einer internen Verwaltungsstruktur und die Entwicklung von Plänen für eine Reorganisation der Abteilung voranzutreiben.

Als im Dezember die Aufgaben des gesamten OSS in der Direktive JCS 155/4/D klar definiert wurden, stand der Verwirklichung dieser Planungen nichts mehr entgegen $^{37}$. Im Zuge der nun durchgeführten Reorganisation wurden mit den Abteilungen Europe-Africa, Far East, USSR und Latin America vier, jeweils für eine Region zuständige "Divisions" geschaffen. Die bislang eigenständigen funktionalen Abteilungen wurden als Economic Capabilities Sub-Division und Topographic Intelligence-Sub-Division in diese neuen Abteilungen integriert, während das bisherige Personal der DSI und der Psychology Division den Mitarbeiterstab für die jeweils neu eingerichteten Psychological Warfare Sub-Divisions bzw. die später daraus hervorgehenden Political Subdivisions stellte. Da der europäische Kriegsschauplatz in der anglo-amerikanischen "Grand Strategy" Priorität hatte ("Germany first“), wurde der Europe-Africa Division dabei das meiste Personal zugeteilt ${ }^{38}$.

Die Europe-Africa Division unterstand der Leitung von Sherman Kent und gliederte sich - ähnlich wie die anderen regionalen Abteilungen - in die wiederum nach Regionen unterteilte Psychological Warfare Sub-Division und die nach thematischen Gesichtspunkten strukturierten Economic Capabilities und Topographic Intelligence Sub-Divisions. Später wurden diese drei Unterabteilungen als Political, Eco-

${ }^{34}$ Dazu und zum Folgenden die Unterlagen in NA, RG 226, E 145, b 2, f 13 und 24; b 4, f 40 ff., sowie $E 146, b$ 70, f 986 und 990.

35 NA, RG 226, R\&A-History, (III), S. 20.

${ }^{36}$ Ebenda, S.23; zum Folgenden S.21 ff.

37 Dazu OSS War Report I, S. 167 ff.; sowie NA, RG 226, E 1, b 2, Administrative Office: R\&A Administrative Regulation No.1, 21.1. 1943; vgl. außerdem die bei Katz, Foreign Intelligence, im Anhang wiedergegebene Graphik „Organization of the Research and Analysis Branch, OSS" (Chart 2).

${ }^{38}$ OSS War Report I, S. 172. 
nomics und Geographic Subdivision geführt ${ }^{39}$. Das in dieser neuen Organisation liegende Potential für fachübergreifende Zusammenarbeit sollte sich als ein wichtiger Faktor für die Leistungsfähigkeit der Europe-Africa Division erweisen; es wurde erstmals umfassend genutzt, als es für die Mitarbeiter der Abteilung ab Herbst 1943 galt, den Löwenanteil der in Vorbereitung der US-Militäradministration geplanten Civil Affairs Guides auszuarbeiten, denn dieses Projekt forderte und förderte interdisziplinäres Arbeiten ${ }^{40}$.

Zusätzlich zu den im Rahmen dieser Reorganisation geschaffenen vier regionalen Hauptabteilungen wurde mit der Map Division eine neue funktionale Abteilung eingerichtet ${ }^{41}$. Die Aufgaben der in dieser Abteilung beschäftigten Spezialisten für Kartographie und Topographie erschöpften sich keineswegs in der bloßen Illustration von R\&A-Studien durch Karten und Modelle; nicht selten stellten die Arbeiten dieser Abteilung vielmehr hochspezialisierte Studien dar, die durchaus als „intelligence reports in themselves ${ }^{\alpha 2}$ gelten konnten.

Neben diesen im Januar 1943 neu gebildeten Abteilungen blieb die Central Information Division (CID), Anfang 1943 noch unter Leitung von Lewis, bestehen. Sie büßte allerdings im Laufe des Jahres viel von ihrer Leistungsfähigkeit ein ${ }^{43}$, denn ihre Mitarbeiter waren ständig überarbeitet und chronisch unterbezahlt. Als Lewis aufgrund verschiedener Spannungen, aber auch wegen seines nachlassenden Interesses an der Arbeit für CID im Oktober 1943 zurücktrat, bestand ein Rückstau von etwa 50-75000 unbearbeiteten Dokumenten. Der Nachfolger von Lewis, Lieutenant Raymond Deston, im Privatberuf ein erfolgreicher Manager, stellte die Leistungsfähigkeit der Abteilung jedoch bald wieder her, und 1944 wurden in der CID pro Monat durchschnittlich etwa 10000 Dokumente bearbeitet.

Bei diesen Dokumenten handelte es sich sowohl um klassifizierte wie frei zugängliche Informationen, die entweder vom OSS selbst gesammelt oder von anderen Behörden zur Verfügung gestellt wurden ${ }^{44}$. Besonders die Beschaffung klassifizierter Materialien war allerdings, wie bereits angedeutet, nicht immer einfach, was der OSS War Report der "natural reluctance of established agencies to recognize the over-all nature of a new organization such as R\&A" zuschreibt ${ }^{45}$. Bis Anfang 1942 hatte R\&A in Roosevelts Sohn, dem Marineoffizier James Roosevelt, jedoch genau den richtigen Verbindungsoffizier, um diesem "natürlichen Widerstreben“ entgegenzutreten. Roosevelt, der auf Ersuchen Donovans im August 1941 von der Navy für die Arbeit beim COI freigestellt worden war, wurde jedoch nach dem japanischen Angriff auf Pearl Harbor

39 Vgl. NA, RG 226, E 1, b 1, f 36: Joint Security Control: Preliminary List of Members of the R. and A. Branch for Whom Authorization to see Top Secret Documents is Asked [o.D.]; sowie ebenda, b 3, f: Europe-Africa Division 1945: Personalliste vom 20.10. 1945; vgl. auch die bei Katz, Foreign Intelligence, im Anhang wiedergegebene graphische Darstellung der Organisation der R\&A-Branch (Chart 2).

40 Ausführlich dazu unten V., 1. Teil.

41 Zur Arbeit der Map Division siehe OSS War Report I, S.169f;; NA, RG 226, R\&A-History, (f 46), Map Division.

42 OSS War Report I, S. $169 \mathrm{f}$.

43 Zum Folgenden ebenda, S.169, und NA, RG 226, R\&A-History, (CID), S.12ff.

44 OSS War Report I, S. 169, vgl. außerdem Katz, Foreign Intelligence, „Flow of R\&A Intelligence Material“ (Anhang: Chart 3).

45 OSS War Report I, S.173. 
zum aktiven Dienst einberufen ${ }^{46}$, und sein Weggang Anfang 1942 bedeutete für R\&A einen empfindlichen Verlust. Dies galt um so mehr, als zu dieser Zeit auch gerade die Secret Intelligence-Branch (SI) des COI aufgebaut wurde, die dazu überging, „to take (and keep) most of the incoming material brought by his successor " ${ }^{\text {47 }}$. Zwar wurde in der CID eigens ein Stab eingerichtet, dessen Aufgabe es war, einen möglichst reibungslosen Austausch von Informationen innerhalb des OSS und mit anderen Behörden zu gewährleisten, doch funktionierte dies niemals zur vollen Zufriedenheit von R\&A ${ }^{48}$. Eine Ausnahme bildete die allerdings auch nicht völlig ungetrübte Zusammenarbeit mit dem State Department ${ }^{49}$, das R\&A regelmäßig Paraphrasen eines Großteils der für die Arbeit der Abteilung relevanten Kabel zugänglich machte.

Die Tätigkeit der CID erschöpfte sich jedoch nicht in der Beschaffung und Bearbeitung von aktuellen Meldungen. Die CID unterhielt beispielsweise auch eine eigene Bibliothek ${ }^{50}$, in der als Leihgaben der Library of Congress von R\&A-Mitarbeitern häufig benötigte Werke bereitstanden. Darüber hinaus enthielt die CID-Bibliothek „special collections" wie etwa die im Frühjahr 1942 in New York konfiszierten Bestände der "German Library of Information", einer nationalsozialistischen Propagandaorganisation $^{51}$, und des „German-American Board of Trade“. Zu einer weiteren wichtigen CIDAbteilung entwickelte sich die ursprünglich zur SI-Branch gehörende Biographical Records Section ${ }^{52}$. Nachdem ihre Mitarbeiter zunächst ohne klare Leitlinien wenig erfolgreich experimentiert hatten, wurde diese Abteilung der CID angegliedert und unter Beteiligung von Hajo Holborn, Felix Gilbert, Eugene Anderson sowie der Leiterin der New Yorker Außenstelle von Biographical Records, Emmy Rado, reorganisiert. Aufgabe der Abteilung war die Sammlung und Überprüfung biographischer Informationen sowie der Aufbau von Karteien über Personen, die in Deutschland und im besetzten Europa politische Funktionen ausübten oder anderweitig über Einfluß verfügten.

Neben der CID wurde Ende April 1943 noch ein Stab eingerichtet, dessen Aufgabe es war, "to set significant current intelligence in a brief, accurate framework of the research analysts' comments or reservations and get the complete intelligence report to policy-level users as quickly as possible ${ }^{\text {ct53 }}$. Der Kern dieses Current Intelligence Staff

${ }^{46}$ Ebenda, S. 11, 20.

47 NA, RG 226, R\&A-History, (CID), S. 12.

$48 \mathrm{Vgl}$. dazu auch OSS War Report I, S.173f.

49 Zum Folgenden ebenda, S. 169; NA, RG 226, R\&A-History, (CID), S. 42 f.; NA, RG 226, E 1, b 2, f: State Department 1944, Holmes an Riddell, 24.8. 1944; vgl. dazu auch Cline, Secrets, S. $42 \mathrm{f}$.

${ }^{50}$ Zum Folgenden NA, RG 226, R\&A-History, (CID), S. $18 \mathrm{ff}$.

${ }^{51}$ Dazu der 68 Seiten umfassende "Index of the German Library of Information" in NA, RG 59, R\&A 427.

52 Zum Folgenden OSS War Report I, S.169; NA, RG 226, R\&A-History, (CID), S. 45 ff.; RG 226, E 1, b 1, f 20 und b 2, f: CID, Biographical Records Section, 1944, siehe insbesondere Field Unit, N.Y.C. of Biographical Records - Report on First Year Activity, June 7, 1943 June 7, 1944.

53 NA, RG 226, R\&A-History, (CIS), S. 6; zum Folgenden auch OSS War Report I, S. 172f.; NA, RG 226, R\&A-History, (CIS), S. 1 ff. Einer der spektakulärsten Erfolge des CIS war die prompte Ausarbeitung einer Serie von Berichten zu dem am 20.Juli 1944 in Deutschland versuchten Staatsstreich, vgl. NA, RG 226, R\&A-History, (CIS), S. 12; ausführlicher dazu unten IV., 3. Teil; zur Arbeit des CIS vgl. auch Cline, Secrets, S. $55 \mathrm{ff}$. 
(CIS) bestand anfänglich aus den Mitarbeitern des Büros Donald McKays, das die beiden OSS-Publikationen The War This Week und OSS Weekly Summary herausgegeben hatte. Der Historiker S. Everett Gleason, der später zusammen mit Langer einige voluminöse Studien über die Vorkriegsjahre in den USA verfassen sollte, übernahm die Leitung dieses Teams, und die R\&A-History bescheinigt ihm „extraordinary energy and imagination in coordinating and extending the staff's operations "54. Für die erfolgreiche Arbeit dieses Stabes war allerdings auch das Wohlwollen General Magruders unverzichtbar. Er hatte die Tätigkeit von SI, R\&A und FN zu überwachen und ermöglichte es den CIS-Mitarbeitern, viele Dokumente - nicht zuletzt manche der von SI eifersüchtig gehüteten „intelligence cables" - auf unbürokratischem und somit schnellem Wege zu erhalten.

Auf Anregung und mit Unterstützung General Magruders gab der CIS auch die für den OSS-internen Gebrauch bestimmte Daily Intelligence Summary heraus ${ }^{55}$. Außer Top-Secret-Informationen enthielt diese Zusammenstellung alle dem OSS verfügbaren aktuellen Informationen, weswegen sie aus Sicherheitsgründen nur in beschränktem Umfang verteilt werden konnte. Daneben gab der CIS-Mitarbeiter Arthur M. Schlesinger das ebenfalls für den OSS-internen Gebrauch bestimmte Psychological Warfare Weekly heraus ${ }^{56}$, das sich offenbar großer Beliebtheit erfreute, was nach Darstellung der R\&A-History in erster Linie „Schlesinger's peppy style and all-consuming intellectual appetite " 57 zu verdanken war. Als Schlesinger im Sommer 1944 nach Europa ging, wurde dieses Magazin durch das etwas strenger gehaltene Political Intelligence Weekly ersetzt. Der CIS vertrat das OSS außerdem im Editorial Committee des Joint Intelligence Committee und steuerte etwa zwei Drittel zu dessen für 65 Spitzenpolitiker bestimmter Publikation JIC Weekly Summary bei ${ }^{58}$. Insbesondere die Beiträge zu politischen und wirtschaftlichen Themen waren im allgemeinen R\&A-Studien entnommen, und die R\&A-History schreibt diesen Erfolg vor allem dem von Gleason eingeführten „careful standard of objectivity in intelligence writing “ $\mathrm{zu}^{59}$.

Nach dieser umfassenden Reorganisation im Januar 1943 wurde die Arbeit der R\&A-Branch von einem Projects Committee geleitet ${ }^{60}$. Dieses Komitee war zunächst als Unterausschuß des Board of Analysts eingerichtet worden; es entschied über die Arbeitsvorhaben der verschiedenen R\&A-Abteilungen, edierte fertige R\&A-Studien und sorgte für ihre Verteilung innerhalb des OSS sowie an die jeweils interessierten Washingtoner Behörden. Das Projects Committee verbesserte die unzureichende Leitung der Arbeit von R\&A durch das Board of Analysts entscheidend; überhaupt konn-

${ }^{54}$ NA, RG 226, R\&A-History, (CIS), S.3.

55 NA, RG 59, R\&A 1142.1 (-.750), „Psychological Warfare Daily Intelligence Summary“ (und andere 'Titel, etwa „The Daily Intelligence Summary“), 22.5. 1943-15.11.1945.

56 NA, RG 59, R\&A 1150.1 (-.52), „The PW Weekly“, 9.4. 1943-28.4. 1944.

57 NA, RG 226, R\&A-History, (CIS), S. 16.

${ }^{58}$ OSS War Report I, S. 173.

59 NA, RG 226, R\&A-History, (CIS), S. 15.

${ }^{60}$ Dazu und zum Folgenden OSS War Report I, S. 168, $171 \mathrm{f}$.; die täglich stattfindenden Sitzungen des Projects Committee sind dokumentiert in NA, RG 226, E 59: Research and Analysis Branch, Projects Committee, Minutes of Meetings, Aug 1942 - Jan 1946; außerdem: NA, RG 226, E 99, b 104, R\&A 2405, „The Work of the Projects Committee“, 1.8. 1944; sowie NA, RG 226, E 37, b 5, f: Projects Committee Correspondence (1943-1945). 
ten durch die Reorganisation der Abteilung wesentliche Probleme gelöst werden: Die neue abteilungsinterne Administration wurde allgemein als Verbesserung empfunden, vor allem aber war die hinderliche Trennung zwischen regionalen und funktionalen Abteilungen aufgehoben und eine Struktur geschaffen worden, die der fachübergreifenden Arbeitsweise, die viele der von R\&A bearbeiteten Fragestellungen erforderten, sehr viel zuträglicher war.

Manche Probleme ließen sich durch diese Reorganisation allerdings nicht lösen und beeinträchtigten die Arbeit von R\&A weiterhin spürbar. Vor allem wurde der bereits angesprochene „Prestigeverlust“ der R\&A-Branch innerhalb des OSS und die daraus resultierende Vernachlässigung der Abteilung von R\&A-Mitarbeitern immer wieder beklagt. Die Palette dieser Klagen reichte von Beschwerden über einen Mangel an wissenschaftlichen Mitarbeitern, Schreibkräften und Räumlichkeiten bis hin zu der Kritik, $\mathrm{daß}$ es manche OSS-Abteilungen - vornehmlich SI - an der notwendigen Kooperation fehlen ließen ${ }^{61}$, wobei die wegen des Krieges besonders strengen Sicherheitsbestimmungen offenbar nicht selten als "the perfect excuse for uncooperativeness" 62 herhalten mußten. Überdies verschärfte sich der schon lange beklagte Personalmangel im Laufe des Krieges durch Einziehung von R\&A-Mitarbeitern zum Militär so sehr, daß das Projects Committee im Februar 1943 Veranlassung sah, die Frage zu erörtern, wie lange die Arbeit von R\&A unter diesen Umständen noch fortgesetzt werden könn$\mathrm{te}^{63}$. Zwar wurde immer wieder von der Möglichkeit Gebrauch gemacht, als besonders unabkömmlich geltende Mitarbeiter vom Militärdienst freizustellen oder ihnen zu erlauben, in Uniform zu ihrer Arbeit bei R\&A zurückzukehren, doch waren solche Ausnahmeregelungen stets "distasteful and difficult to obtain"64. In zunehmendem Maße sah sich R\&A überdies durch die starke Inanspruchnahme der Abteilung bei der Planung und Vorbereitung der amerikanischen Besatzungs- und Nachkriegspolitik belastet. Insbesondere das im Sommer 1943 unter Leitung von Hajo Holborn entwickelte Civil Affairs Guide-Programm erwies sich als ungeheuer arbeitsintensiv und problematisch ${ }^{65}$.

Gleichzeitig wurde R\&A-Personal von der Washingtoner Zentrale auf OSS-Außenposten abgezogen ${ }^{66}$. Die erste COI-Außenstelle war bereits im Oktober 1941 in Lon-

${ }^{61}$ Vgl. NA, RG 226, E 1, b 1, f 5: Memorandum vom 17.9. 1943, und ebenda, f 38: Schreiben an Langer vom 7.6. 1944; über die mangelnde Kooperationsbereitschaft sowie die "Geheimniskrämerei" von SI wurde - besonders im Herbst 1943 - mehrfach Klage geführt, vgl. dazu auch Winks, Cloak, S.76f. Die unzulängliche Kooperation innerhalb des OSS und zwischen den US-Nachrichtendienststellen im allgemeinen illustriert auch eine Auflistung der EuropeAfrica Division über „Intelligence Requirements in Respect to Germany" vom 19.10.1943 in: NA, RG 59, R\&A 1531; zum akuten Personalmangel in R\&A vgl. außerdem die in NA, RG 226, E 42 (1 box) enthaltenen Monthly Progress Reports der Europe-Africa Division; R\&ASchreibkräfte brachten es demnach bspw. auf bis zu 70 Überstunden pro Monat.

62 NA, RG 226, R\&A-History, (III), S.23.

${ }_{63}$ NA, RG 226, E 59, II, Sitzung vom 8.2. 1943.

${ }^{64}$ OSS War Report I, S.168.

${ }^{65}$ Dazu ausführlich unten V., 1. Teil.

${ }^{66}$ Zum Folgenden NA, RG 226, R\&A-History, (f 45), S. 105 ff.; OSS War Report I, S.170f. und II, S.259ff.; sowie die Unterlagen in NA, RG 226, E 50, Research and Analysis Branch, Europe-Africa Division, Records Relating to Outposts in Germany 1945; ausführlicher dazu unten VI., 1. Teil. 
don eingerichtet worden ${ }^{67}$. Wie Präsident Roosevelt am 24. Oktober in einem Schreiben an Churchill darlegte, war Donovan autorisiert worden, „to send a small staff to London"; die Aufgabe dieses Außenpostens sollte es sein, die europäische Länder betreffende Arbeit des COI zu erleichtern. Daß dieser „small staff “ bis zum Frühjahr 1944 auf über 2000 Mitarbeiter anwuchs, illustriert die Bedeutung dieser Außenstelle. Mitte Mai 1942 war in London auch eine R\&A-Abteilung eingerichtet worden ${ }^{68}$, die sich nach anfänglichen Schwierigkeiten zu einem wichtigen - wenn auch im eigenen Verständnis von Washington nicht immer entsprechend gewürdigten - Partner bei den Bemühungen um eine sinnvolle Planung und Vorbereitung der amerikanischen Besatzungspolitik in Deutschland entwickelte und die Zusammenarbeit mit in London stationierten alliierten Behörden wie etwa der European Advisory Commission (EAC) erleichterte.

Im Verlaufe des Krieges wuchs die Bedeutung dieser Außenstellen für die Arbeit von R\&A; und besonders nach der Invasion Europas stellten das Londoner R\&A-Büro sowie die den Truppen unmittelbar folgenden OSS-Teams die Versorgung der Washingtoner Zentrale mit aktuellen Informationen und Dokumentationsmaterialien sicher. Im September 1944 wurde auch in Paris ein OSS-Büro eingerichtet, und als US-Streitkräfte begannen, in Deutschland einzurücken, folgten ihnen R\&A-Teams, „to undercover Nazi officials and hidden assets and to provide basic intelligence for the U.S.Group of the Control Council" ${ }^{\text {699 }}$.

Insgesamt sollte sich R\&A so trotz der eben beschriebenen Widrigkeiten letztlich zum "most respected part of the entire OSS operation" entwickeln ${ }^{70}$. Die Abteilung produzierte bis Kriegsende etwa 3000 Studien, deren Umfang von manchmal nur knapp eine Seite umfassenden Kurzberichten zu bestimmten Fragestellungen bis zu massiven Werken wie etwa dem für die Besatzungstruppen ausgearbeiteten, rund 1500 Seiten umfassenden Handbuch zu Süddeutschland reichte ${ }^{71}$. Daneben wurde eine Materialsammlung aufgebaut, die auf 3 Millionen Karteikarten, 300000 Photos, ebenso viele „intelligence documents“, 1 Million Karten, 350000 Ausgaben ausländischer Serienpublikationen, 50000 Bücher, Tausende von biographischen Akten und Tausende von Postkarten geschätzt worden ist ${ }^{72}$. Angesichts dieser Zahlen mag der Verdacht naheliegen, daß die Qualität der von R\&A geleisteten Arbeit unter deren Quantität litt. Doch auch wenn die von der Abteilung ausgearbeiteten Studien nicht frei von Fehlern und Irrtümern waren, und auch wenn sich einzelne Beispiele finden ließen, die im Rückblick gar als Makulatur erscheinen mögen, bezeugen die R\&A-Arbeiten doch in

${ }^{67}$ Dazu und zum Folgenden OSS War Report I, S.15; OSS War Report II, S. 3-7; vgl. auch Troy, Donovan, S. 115, 117, der darauf hinweist, daß die Einrichtung dieser ersten COI-Außenstelle anfangs von den Washingtoner Querelen um den COI überschattet war.

68 NA, RG 226, E 91, b 7-10, War Diary, R\&A-Branch, OSS London (im folgenden zitiert als R\&A/London, War Diary), Vol. I: Early History; zum Folgenden Vol. VIII: Civil Affairs, sowie Vol. III (1944).

${ }^{69}$ OSS War Report II, S. 327; ausführlicher dazu unten VI., 1. Teil.

70 Corson, Armies of Ignorance, S.168; ähnlich Winks, Cloak, S.61; Troy, Donovan, S. 85; Stephenson, zit. in Dunlop, Donovan, S. 309.

71 NA, RG 59, R\&A 2332, „South Germany“, 22. 9. 1944; vgl. dazu auch Corson, Armies of Ignorance, S.173; Winks, Cloak, S. 111.

72 Ebenda, S.110f. 
ihrer großen Mehrheit die bemerkenswerte Leistungsfähigkeit der Abteilung und die überragende fachliche Kompetenz ihrer Mitarbeiter ${ }^{73}$.

Auf einem anderen Blatt steht freilich die Frage, welcher Nutzen aus diesen Arbeiten tatsächlich gezogen wurde. Obwohl sich aus abteilungsinternen Unterlagen entnehmen läßt, daß R\&A-Materialien jeden Monat in hundert- oder gar tausendfacher Vervielfältigung in Washington verteilt wurden ${ }^{74}$, erlaubt dies kaum Rückschlüsse darauf, ob und inwieweit die darin enthaltenen Informationen, Analysen und Empfehlungen letztlich auch rezipiert wurden ${ }^{75}$. Zwar finden sich in den Unterlagen der Abteilung zuweilen Hinweise auf positive Reaktionen von R\&A- „Kunden“, und gelegentlich werden einer Studie konkrete Auswirkungen zugeschrieben ${ }^{76}$; hin und wieder werden auch kritische Stellungnahmen diskutiert, doch sind diese Hinweise insgesamt zu unvollständig - und im übrigen kaum nachprüfbar -, als daß sich darauf ein verläßliches Urteil gründen ließe.

${ }^{73}$ Die wenigen Historiker, die sich bislang eingehender mit der Arbeit von R\&A befaßt haben, stimmen in diesem Urteil im wesentlichen überein, siehe dazu generell Katz, Foreign Intelligence, und Winks, Cloak; außerdem Corson, Armies of Ignorance, S. 168ff. Smith, Shadow Warriors, S. 360 ff., äußert sich zuweilen zwar kritisch oder auch spöttisch, zeichnet aber insgesamt doch ein positives Bild der von R\&A geleisteten Arbeit.

${ }^{74}$ Dazu insbesondere NA, RG 226, E 42 (1 box): R\&A, Europe-Africa Division, Monthly Progress Reports, Sept. 1943 - June 1945, sowie NA, RG 226, E 59: Research and Analysis Branch, Projects Committee, Minutes of Meetings, Aug 1942 - Jan 1946. Der Rechenschaftsbericht für März 1945 (E 42) verzeichnet z. B. 73 fertiggestellte und vom Projects Committee gebilligte Studien (davon waren 10 von den JCS, 13 vom State Department, 13 vom War Department und weitere 7 von anderen Behörden angefordert worden; der Rest dürte auf OSS- oder R\&A-interne Initiative zurückgehen); 62 neue Projekte werden vom Projects Committee genehmigt (davon waren 5 von den JCS, 11 vom State Department und 7 von anderen Behörden angefordert worden). Außerdem wird die Verteilung von 10365 Kopien von R\&A-Berichten verzeichnet (3450 in Washington, davon 594 an das War Department, 123 an das Navy Department, 785 an das State Department, 1394 innerhalb des OSS und 554 an verschiedene andere Behörden). Des weiteren werden Beiträge zu OSS-internen Informationsmaterialien sowie zu der vom Joint Intelligence Committee wöchentlich für Spitzenpolitiker und Ministerialbeamte herausgegebenen Zusammenfassung aktueller Meldungen verzeichnet; auBerdem seien zahlreiche „special memoranda on highest priority OSS intelligence for immediate high-level distribution" ausgearbeitet und folgendermaßen verteilt worden: Präsident 25, JCS - 19, Secretary of State - 25, Assistant Secretary of State 8, sowie einzelne Meldungen an verschiedene andere Stellen. - Für eine Auswertung dieser Zahlen ist problematisch, $\mathrm{da} ß$ sie nicht $\mathrm{zwischen}$ den von R\&A ausgearbeiteten Analysen einerseits und andererseits $\mathrm{Be}-$ richten oder Meldungen unterscheiden, die von R\&A- bzw. CID-Mitarbeitern lediglich überprüft und eventuell zu Kurzberichten ausgearbeitet oder mit einem Kommentar versehen wurden.

75 Vgl. dazu auch die Überlegungen bei Smith, Shadow Warriors, S. $373 \mathrm{ff}$ - Katz, Foreign Intelligence, $x i$ ff., sieht die Verdienste von R\&A "less in the field of diplomatic or military history than in intellectual history " und konzentriert sich in seiner Darstellung auf diesen Bereich. Winks, Cloak, S.112ff., meint zwar, es gebe „little historical disagreement that R\&A was the most important unit in the OSS“, vertritt aber im übrigen die Ansicht: „One simply cannot know what effect these [... R\&A] reports had on the war."

${ }^{76}$ Vgl. etwa den oben, II., 1. Teil, erwähnten Rechenschaftsbericht der Economics Division; auBerdem: „Achievements of the Research and Analysis Branch During the Past Year", 23.4. 1945, in: NA, RG 226, E 99, b 104; sowie "Contributions in the Field of Social Sciences made by the Research and Analysis Branch, OSS, to U.S. Strategic Intelligence“, in: NA, RG 226, E 37, b 5, f: R\&A History, Experiences, etc. 
Andererseits deutet aber der Umstand, daß die JCS sowie die wichtigsten Washingtoner Ministerien - insbesondere das State Department - regelmäßig R\&A-Studien in Auftrag gaben, zumindest auf deren ernsthaftes Interesse an der Arbeit der Abteilung. Auch die Tatsache, daß R\&A nach Kriegsende nicht wie die übrigen OSS-Abteilungen aufgelöst wurde, sondern im State Department den Kern eines neu aufzubauenden Auslandsnachrichtendienstes bilden sollte 7 , spricht dafür, daß sich die von der Abteilung geleistete Arbeit in Washington einer nicht unbeträchtlichen Wertschätzung erfreute. Obwohl diese "Indizien" kaum "definitive answers" erlauben mögen ${ }^{78}$, deuten sie doch darauf hin, daß durch die bisherige Vernachlässigung der Arbeit der R\&ABranch in der Historiographie ein ernstzunehmender Aspekt des "Know-how" amerikanischer Behörden in der Kriegs- und Nachkriegszeit unberücksichtigt geblieben ist.

77 Ausführlicher dazu unten VII., 1. Teil.

78 "Those who require definitive answers ought not to read history " so der Yale-Historiker Winks, Cloak, S.110, zur Frage nach der praktischen Bedeutung der von R\&A geleisteten Arbeit. 